\title{
Young, male, road traffic victims: a systematic review of the published trauma registry literature from low and middle income countries
}

\author{
Oliver Boughton ${ }^{1, *}$, Gareth G. Jones ${ }^{1}$, Christopher B.D. Lavy ${ }^{2}$, and Caris E. Grimes ${ }^{3}$ \\ 1 The MSk Lab, Imperial College London, London SW7 2AZ, UK \\ 2 Nuffield Department of Orthopaedics, Rheumatology and Musculoskeletal Sciences, Oxford University, Oxford OX1 2JD, UK \\ 3 King's Centre for Global Health, King's College London, London WC2R 2LS, UK
}

Received 22 February 2015, Accepted 16 April 2015, Published online 15 June 2015

\begin{abstract}
Background: Trauma contributes significantly to the global burden of disease. We analysed published trauma registries to assess the demographics of those most affected in low and middle-income countries (LMICs). Methods: We performed a systematic review of published trauma registry studies according to PRISMA guidelines. We included published full-text articles from trauma registries in low and middle-income countries describing the demographics of trauma registry patients. Articles from military trauma registries, articles using data not principally derived from trauma registry data, articles describing patients of only one demographic (e.g. only paediatric patients), or only one mechanism of injury, trauma registry implementation papers without demographic data, review papers and conference proceedings were excluded.

Results: The initial search retrieved 1868 abstracts of which 1324 remained after duplicate removal. After screening the abstracts, 78 full-text articles were scrutinised for their suitability for inclusion. Twenty three papers from 14 countries, including 103,327 patients, were deemed eligible and included for analysis. The median age of trauma victims in these articles was 27 years (IQR 25-29). The median percentage of trauma victims who were male was 75 (IQR 66-84). The median percentage of road traffic injuries (RTIs) as a percentage of total injuries caused by trauma was 46 (IQR 21-71).

Conclusions: Young, male, road traffic victims represent a large proportion of the LMIC trauma burden. This information can inform and be used by local and national governments to implement road safety measures and other strategies aimed at reducing the injury rate in young males.
\end{abstract}

Key words: Trauma registry, Low and middle-income countries.

\section{Introduction}

In 2010 there were 5.1 million worldwide deaths attributable to injury. This accounts for $9.6 \%$ of all global deaths and has been increasing over the last 20 years [18]. To put this in context, injuries account for more deaths than HIV-AIDS (human immunodeficiency virus-acquired immune deficiency syndrome), tuberculosis and malaria combined [24]. In low and middle-income countries (LMICs) there is a greater toll of injury than high-income countries [40], with $90 \%$ of world deaths resulting from injury occurring in LMICs [49]. Injury can also result in lifelong disability [49], with significant financial implications for the injured patient and their family [42].

*Corresponding author: o.boughton@imperial.ac.uk
Injuries disproportionately affect males and the young $[11,24]$.

As a subgroup of injuries, road traffic injuries (RTIs) are the leading injury-related cause of death in males and were the ninth leading cause of death worldwide in 1999 [31]. RTIs accounted for $14 \%$ of deaths in males aged 10-24 years and $5 \%$ of female deaths in the same age group in 2004 [30]. In 2010, RTIs accounted for 1.3 million deaths worldwide and there was a $46 \%$ increase in death due to RTIs compared to two decades earlier [18]. Whereas deaths in high-income countries with road safety programmes have reduced over the last few years, deaths from RTIs in LMICs have increased [18]. RTIs are predicted to become the third or fourth leading cause of death in the world by 2030 [19].

A trauma registry may be defined as "a timely, accurate, and comprehensive data source that allows for continuous 
monitoring of the process of injury care" [26]. The data encompasses all hospital trauma-related admissions and is a powerful tool for identifying injury trends and possible solutions [4, 29]. Trauma registry data are particularly valuable in LMICs because other sources of data, which might be available in high-income countries, are less accessible in LMICs [25].

We set out to use published trauma registry data from LMICs to determine the current demographics of trauma patients in LMICs, as a basis for the development of intervention strategies. Specifically, we wanted to answer the questions:

1. Do young, male patients continue to be most affected by trauma?

2. How much do RTIs contribute to the burden of trauma in LMICs?

To answer these questions we performed a systematic review of the published trauma registry literature from LMICs.

\section{Method}

We performed a systematic review of the published trauma registry literature from LMICs. Medline, Embase, Cochrane Library, PubMed, CINAHL and Web of Science from design to the 30th May 2014 were searched using single and combinations of the search terms "developing world", "developing country", "low income country", "middle income country", "trauma database/databank", "trauma registry/registries", "injury database/databank" and "injury registry/registries". We included published full-text articles from trauma registries in low and middle-income countries (as defined by the World Bank [1]) that describe the demographics of their trauma registry patients. Authors were contacted by email if full-text articles were unavailable. Articles from military trauma registries were excluded on the basis that their patient demographics and mechanisms of injury would be different. Articles from highincome countries, articles using data not principally derived from a trauma registry, articles describing patients of only one demographic (e.g. only paediatric patients) or only one mechanism of injury (e.g. only RTIs) were excluded from the final analysis. Trauma registry implementation or design papers, review papers and conference proceedings were excluded. Two authors selected articles for the qualitative and quantitative analyses and disagreements about whether a study should be included were resolved by discussion, as advised by the Cochrane Collaboration [10].

For the quantitative analysis, articles that used the same data from another article were not included. For example, if there were two articles published from the same trauma registry data of the same or similar years, only one of the articles would be chosen for quantitative analysis. During the qualitative analysis if data from an article were decided to not be trauma registry data, if the data only represented one patient group (e.g. one age group of patients) or if the data were incomplete, the article would be excluded from the quantitative analysis. Data from the included articles were analysed using
IBM SPSS Statistics version 22. Average patient age, gender and mechanism of injury were analysed. Additional data on method and time of pre-hospital transfers were analysed, if available. Medians and interquartile ranges were chosen to represent the results, as the data distribution was non-parametric. If an article reported the average age of their patients as both a median and a mean, the median value was chosen for the purpose of analysis. If, however, only a mean was reported, the mean was accepted and used for the analysis. An assessment of the quality of articles was made, based on the "Trauma Registry Assessment Tool" designed by O'Reilly et al. [28].

\section{Results}

Figure 1 shows the systematic review flowchart according to PRISMA guidelines for systematic reviews [21]. The search retrieved 1867 abstracts from database searching and one additional record from a reference in an article's bibliography. Abstracts (1324) remained after duplicates were removed and 78 full-text articles were reviewed after abstract screening. Twenty three papers from 14 countries, including 103,327 patients, were deemed eligible and included in the qualitative analysis. Table 1 displays the articles included in the qualitative analysis. Sixteen of these articles were included in the quantitative analysis. The explanations for why seven articles were not included in the quantitative analysis are contained in the table. Table 2 displays an assessment of the quality of articles in the qualitative analysis. We made an overall subjective assessment of the quality of the articles retrieved by comparing a trauma registry article to what O'Reilly et al. recommend a trauma registry should report using their "Trauma Registry Assessment Tool" [28].

Table 3 displays the quantitative synthesis of the review. The median age of LMIC trauma victims in this analysis was 27 (IQR 25-29). The median percentage of trauma victims who were male was 75 (IQR 66-84). The median percentage of RTIs as a percentage of total injuries caused by trauma was 46 (IQR 21-71). The median percentage of penetrating injuries (stabbings and gunshots) as a percentage of total injuries caused by trauma was 10 (IQR 4-21). The median percentage of blunt force injuries as a percentage of total injuries caused by trauma was 1 (IQR $0-15$ ). The median percentage of falls as a percentage of total injuries caused by trauma was 17 (IQR 8-31). We found four of the articles in the quantitative synthesis of the review used the "Kampala Trauma Score" [13] to calculate the severity of injuries in their patients. Other trauma scoring systems used included the "Abbreviated Injury Scale" (AIS), the "A Severity Characterization Of Trauma" (ASCOT) score, the Glasgow Coma Scale, the "Injury Severity Score", the "Revised Trauma Score" and the "Trauma and Injury Severity Score".

Table 4 displays the pre-hospital transfer methods and transfer times. Only a few of the articles reported this data. The median transfer time to hospital was 180 min with a large range of transfer times. Pre-hospital transfer methods varied largely between countries and between the articles. The median percentage of ambulance transfers as a percentage of total 


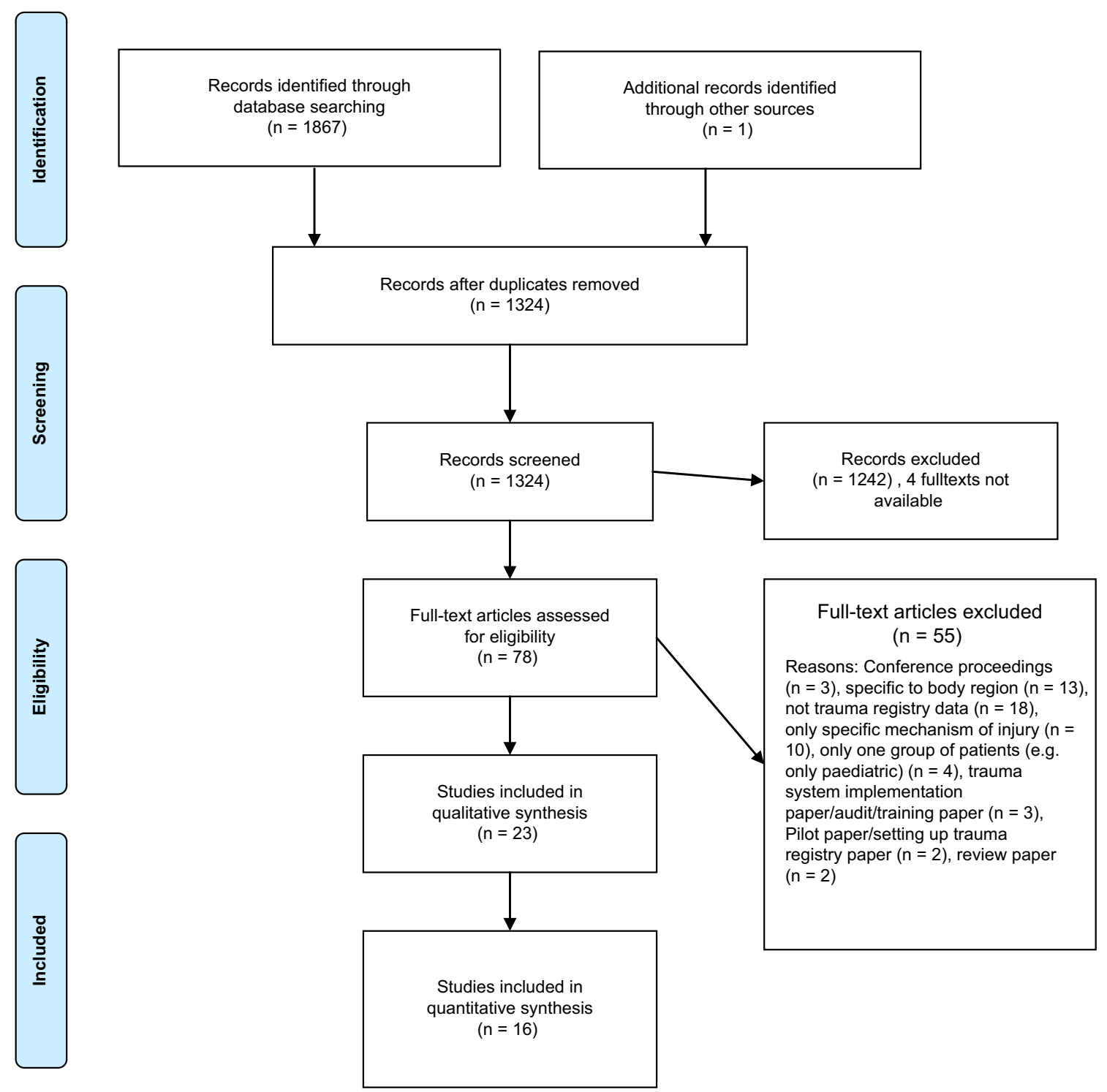

Figure 1. Systematic review flowchart, using PRISMA guidelines for systematic reviews.

pre-hospital transfers was 6 (IQR 5-35). The median percentage of private vehicle transfers as a percentage of total pre-hospital transfers was 44 (IQR 0-52). Other less common methods of pre-hospital transfer included walking, taxi, public transport and police.

\section{Discussion}

This systematic review of published trauma registry data demonstrates that young, male, road traffic victims represent a large proportion of the LMIC trauma burden. These findings are consistent with a previous systematic analysis of the global burden of disease in young people, which found that RTIs accounted for the most disability-adjusted life years (DALYs) in young males aged 10-24 years [7].

Amongst the global population of all ages RTIs accounted for 75.5 million DALYs in 2010, an increase from 56.7 million in 1990 [23]. RTIs accounted for 53\% more of the global burden of disease than tuberculosis in 2010 [23]. Despite this burden, the epidemic of injuries has been described as being "among the most neglected health problems of the late 20th century" [46] with relatively little research conducted into road safety injuries compared to other leading causes of disease [16]. Indeed, investment in injury has fallen behind investments in HIV/AIDS and reproductive health [7]. It is estimated that if injury mortality rates from all causes of injury in LMICs were reduced to those rates seen in high-income countries, over two million lives could be saved each year [15]. Financially, RTIs are estimated to cost LMICs 100 billion US dollars per year according to the World Bank [45], representing 1-3\% of their gross national product (GNP) [36].

Improved road safety programmes can result in dramatic reductions in road traffic injury rates, as demonstrated in Australia where there was a $43.7 \%$ reduction in road trafficrelated mortality following the introduction of road safety 
Table 1. Articles included in qualitative synthesis of systematic review.

\begin{tabular}{|c|c|c|c|c|c|}
\hline Country & Author, Year & Methodology & $\begin{array}{l}\text { Number } \\
\text { of } \\
\text { hospitals }\end{array}$ & $\begin{array}{c}\text { Number of } \\
\text { patients } \\
\text { (over study period) }\end{array}$ & $\begin{array}{l}\text { Included in } \\
\text { quantitative } \\
\text { analysis }\end{array}$ \\
\hline Columbia & $\begin{array}{l}\text { Ordóñez et al. } \\
2012 \text { [29] }\end{array}$ & $\begin{array}{l}\text { Electronic trauma data capture in } \\
\text { emergency department for trauma } \\
\text { patients. }\end{array}$ & 2 & 3923 (3 months) & Yes \\
\hline Fiji & $\begin{array}{l}\text { Wainiqolo } \\
\text { et al. } 2012 \\
\text { [43] }\end{array}$ & $\begin{array}{l}\text { Paper injury surveillance form } \\
\text { using data captured from medical } \\
\text { notes (inpatients only). }\end{array}$ & 12 & 2233 (1 year) & $\begin{array}{l}\text { No: injury } \\
\text { surveillance data } \\
\text { captured from } \\
\text { inpatient medical } \\
\text { notes only. }\end{array}$ \\
\hline India & $\begin{array}{l}\text { Roy et al. } \\
2010 \text { [37] }\end{array}$ & $\begin{array}{l}\text { Paper trauma checklist on } \\
\text { admission to trauma ward. One } \\
\text { hundered and seventy randomly } \\
\text { selected patients from a total of } \\
454 \text { patients admitted to trauma } \\
\text { ward. Excluded: elderly patients } \\
\text { with an isolated fracture of the } \\
\text { neck of the femur. }\end{array}$ & 1 & 170 (2 months) & Yes \\
\hline Iran & $\begin{array}{l}\text { Haghparast- } \\
\text { Bidgoli et al. } \\
2013[8]\end{array}$ & $\begin{array}{l}\text { Validated trauma questionnaire } \\
\text { completed for all admitted trauma } \\
\text { patients. }\end{array}$ & 14 & 17,753 (5 years) & Yes \\
\hline Iran & $\begin{array}{l}\text { Moini et al. } \\
2000 \text { [22] }\end{array}$ & $\begin{array}{l}\text { Paper trauma registry form } \\
\text { completed for all trauma patients } \\
\text { in emergency department and } \\
\text { followed up daily on the ward. }\end{array}$ & 3 & 2663 (1 year) & $\begin{array}{l}\text { No: Same data as } \\
\text { Rabbani/Moini } \\
\text { paper. }\end{array}$ \\
\hline Iran & $\begin{array}{l}\text { Rabbani and } \\
\text { Moini } 2007 \\
{[34]}\end{array}$ & $\begin{array}{l}\text { Paper trauma registry form } \\
\text { completed for all trauma patients } \\
\text { in emergency department and } \\
\text { followed up daily on the ward. }\end{array}$ & 3 & $\begin{array}{l}4096 \text { (likely over } \\
7 \text { years but not } \\
\text { recorded) }\end{array}$ & Yes \\
\hline Jamaica & $\begin{array}{l}\text { Plummer et al. } \\
2010 \text { [33] }\end{array}$ & $\begin{array}{l}\text { Electronic trauma database. } \\
\text { Patients aged } 25-29 \text { years selected } \\
\text { from the database. }\end{array}$ & 1 & 715 (5 years) & $\begin{array}{l}\text { No: only } 25- \\
29 \text { year olds } \\
\text { included. }\end{array}$ \\
\hline Jamaica & $\begin{array}{l}\text { Ward et al. } \\
2010 \text { [44] }\end{array}$ & $\begin{array}{l}\text { Paper injury surveillance form for } \\
\text { trauma patients on arrival in } \\
\text { hospital or after stabilised if } \\
\text { critically unwell. }\end{array}$ & 9 & 40,563 ( 1 year) & Yes \\
\hline Malawi & $\begin{array}{l}\text { Samuel et al. } \\
2010 \text { [39] }\end{array}$ & $\begin{array}{l}\text { Emergency department trauma } \\
\text { registry form filled out for trauma } \\
\text { patients on arrival and } \\
\text { retrospective review of all hospital } \\
\text { ward admissions, discharges and } \\
\text { report log books. Combined data. }\end{array}$ & 1 & 1474 (6 months) & Yes \\
\hline Malaysia & $\begin{array}{l}\text { Sabariah et al. } \\
2008 \text { [38] }\end{array}$ & $\begin{array}{l}\text { All major trauma patients' details } \\
\text { directly entered into electronic } \\
\text { database. }\end{array}$ & 5 & 933 (1 year) & Yes \\
\hline Nigeria & $\begin{array}{l}\text { Nottidge et al. } \\
2014 \text { [25] }\end{array}$ & $\begin{array}{l}\text { Paper trauma registry forms } \\
\text { obtained prospectively in } \\
\text { emergency department for all } \\
\text { patients with injuries. }\end{array}$ & 1 & 93 (7 weeks) & Yes \\
\hline Pakistan & $\begin{array}{l}\text { Hashmi et al. } \\
2013 \text { [9] }\end{array}$ & $\begin{array}{l}\text { Computerised database of all } \\
\text { activated trauma team calls (dead } \\
\text { on arrival and burns excluded). }\end{array}$ & 1 & 1227 (12 years) & Yes \\
\hline Pakistan & $\begin{array}{l}\text { Mehmood } \\
\text { et al. } 2013 \\
\text { [20] }\end{array}$ & $\begin{array}{l}\text { Electronic trauma registry of all } \\
\text { trauma patients in emergency } \\
\text { department (excluding isolated hip } \\
\text { fractures and dead on arrival). }\end{array}$ & 1 & 542 (3 months) & Yes \\
\hline
\end{tabular}


Table 1. (continued)

\begin{tabular}{|c|c|c|c|c|c|}
\hline Country & Author, Year & Methodology & $\begin{array}{l}\text { Number } \\
\text { of } \\
\text { hospitals }\end{array}$ & $\begin{array}{c}\text { Number of } \\
\text { patients } \\
\text { (over study period) }\end{array}$ & $\begin{array}{c}\text { Included in } \\
\text { quantitative } \\
\text { analysis } \\
\end{array}$ \\
\hline Pakistan & $\begin{array}{l}\text { Zafar et al. } \\
2002 \text { [51] }\end{array}$ & $\begin{array}{l}\text { Initial paper trauma form for all } \\
\text { patients meeting trauma team } \\
\text { activation criteria, converted to } \\
\text { online database. Patients operated } \\
\text { on in other hospitals excluded. }\end{array}$ & 1 & $279(2$ years $)$ & $\begin{array}{l}\text { No: same data as } \\
\text { Hashmi et al.'s } \\
\text { paper. }\end{array}$ \\
\hline Rwanda & $\begin{array}{l}\text { Petroze et al. } \\
2014 \text { [32] }\end{array}$ & $\begin{array}{l}\text { Paper trauma registry forms } \\
\text { completed for all injured patients } \\
\text { transferred from a district hospital, } \\
\text { who died in the emergency } \\
\text { department or admitted due to } \\
\text { injury included. Minor injuries } \\
\text { treated as an outpatient excluded. }\end{array}$ & 1 & 2227 (1 year) & Yes \\
\hline South Africa & $\begin{array}{l}\text { Laing et al. } \\
2014 \text { [17] }\end{array}$ & $\begin{array}{l}\text { Electronic trauma registry. } \\
\text { Inclusion criteria: all trauma- } \\
\text { related admissions, all trauma- } \\
\text { related mortalities. Exclusion } \\
\text { criteria: all orthopaedic trauma } \\
\text { cases managed without co- } \\
\text { supervision or consultation from } \\
\text { trauma surgeons, all trauma cases } \\
\text { managed as outpatients, burns } \\
\text { patients, attempted suicides by way } \\
\text { of poison or caustic substance } \\
\text { ingestion, foreign body ingestion, } \\
\text { inhalation. }\end{array}$ & 2 & 2550 (1 year) & Yes \\
\hline South Africa & $\begin{array}{l}\text { Schuurman } \\
\text { et al. } 2011 \\
{[40]}\end{array}$ & $\begin{array}{l}\text { Paper trauma registry forms filled } \\
\text { out for all trauma patients in } \\
\text { emergency department. }\end{array}$ & 1 & 785 (1 month) & Yes \\
\hline Turkey & $\begin{array}{l}\text { Squyer et al. } \\
2008 \text { [42] }\end{array}$ & $\begin{array}{l}\text { Medical records of all trauma } \\
\text { patients admitted retrospectively } \\
\text { reviewed. Compared to US hospital } \\
\text { trauma registry data. }\end{array}$ & 2 & 506 (1 year) & $\begin{array}{l}\text { No: retrospective } \\
\text { data collection of } \\
\text { admitted patients. } \\
\text { Not trauma } \\
\text { registry. }\end{array}$ \\
\hline Uganda & $\begin{array}{l}\text { Demyttenaere } \\
\text { et al. } 2009 \text { [4] }\end{array}$ & $\begin{array}{l}\text { Paper trauma registry forms } \\
\text { completed for all trauma patients } \\
\text { in emergency department. }\end{array}$ & 1 & 3778 (1 year) & Yes \\
\hline Uganda & $\begin{array}{l}\text { Hsia et al. } \\
2010 \text { [11] }\end{array}$ & $\begin{array}{l}\text { Paper trauma registry forms } \\
\text { completed for all trauma patients } \\
\text { in emergency department. }\end{array}$ & 1 & 3750 (1 year) & $\begin{array}{l}\text { No: same data set } \\
\text { as Demyttenaere } \\
\text { et al.'s paper. }\end{array}$ \\
\hline Uganda & $\begin{array}{l}\text { Kobusingye } \\
\text { and Lett } 2000 \\
{[14]}\end{array}$ & $\begin{array}{l}\text { Paper trauma registry forms } \\
\text { completed for all trauma patients } \\
\text { in emergency department. }\end{array}$ & 2 & $\begin{array}{l}5210 \text { (no study } \\
\text { period available in } \\
\text { paper) }\end{array}$ & $\begin{array}{l}\text { No: no study } \\
\text { period available to } \\
\text { assess if same } \\
\text { patients in } \\
\text { Kobusingye et al. } \\
\text { paper from } 2002 \text {. }\end{array}$ \\
\hline Uganda & $\begin{array}{l}\text { Kobusingye } \\
\text { et al. } 2002 \\
\text { [13] }\end{array}$ & $\begin{array}{l}\text { Paper trauma registry forms } \\
\text { completed for all trauma patients } \\
\text { in emergency department. }\end{array}$ & $\begin{array}{c}5 \\
\text { (citywide) }\end{array}$ & 4359 (1 year) & Yes \\
\hline Zambia & $\begin{array}{l}\text { Seidenberg } \\
\text { et al. } 2014 \\
{[41]}\end{array}$ & $\begin{array}{l}\text { Paper trauma registry forms } \\
\text { completed for patients if they } \\
\text { presented to the Surgical } \\
\text { Emergency Centre with evidence } \\
\text { of injury. Additional data collected } \\
\text { on those brought in dead through } \\
\text { the same Emergency Centre. }\end{array}$ & 1 & 3498 (6 months) & Yes \\
\hline
\end{tabular}


Table 2. Assessment of quality of articles.

\begin{tabular}{|c|c|c|c|c|c|c|}
\hline Author, Year & Data capture & $\begin{array}{c}\text { Reported } \\
\text { completeness of } \\
\text { data }(\%)\end{array}$ & $\begin{array}{c}\text { Data collection } \\
\text { staff }\end{array}$ & $\begin{array}{c}\text { Trauma data } \\
\text { collection } \\
\text { methods }\end{array}$ & $\begin{array}{l}\text { Methods to } \\
\text { optimise data } \\
\text { quality }\end{array}$ & $\begin{array}{c}\text { Overall } \\
\text { subjective } \\
\text { assessment }\end{array}$ \\
\hline $\begin{array}{l}\text { Demyttenaere } \\
\text { et al. } 2009 \text { [4] }\end{array}$ & Prospective & 93.5 & Not mentioned & Paper form & Not mentioned & Good \\
\hline $\begin{array}{l}\text { Haghparast- } \\
\text { Bidgoli et al. } \\
2013 \text { [8] }\end{array}$ & Prospective & Not mentioned & $\begin{array}{l}\text { Trained } \\
\text { physicians }\end{array}$ & $\begin{array}{l}\text { Validated } \\
\text { questionnaire } \\
\text { then data } \\
\text { analysed using } \\
\text { IBM SPSS } \\
\text { Statistics }\end{array}$ & $\begin{array}{l}\text { Trained } \\
\text { physicians doing } \\
\text { data collection }\end{array}$ & Good \\
\hline $\begin{array}{l}\text { Hashmi et al. } \\
2013 \text { [9] }\end{array}$ & Prospective & 90 & Trained personnel & Not mentioned & $\begin{array}{l}\text { Data collection } \\
\text { by trained } \\
\text { personnel }\end{array}$ & Good \\
\hline $\begin{array}{l}\text { Hsia et al. } 2010 \\
\text { [11] }\end{array}$ & Prospective & 93 & $\begin{array}{l}\text { Doctors, nurses } \\
\text { and clinical } \\
\text { officers }\end{array}$ & $\begin{array}{l}\text { Paper form then } \\
\text { entered onto } \\
\text { computer } \\
\text { spreadsheet }\end{array}$ & $\begin{array}{l}\text { Data checked by } \\
\text { Senior Doctor }\end{array}$ & Moderate \\
\hline $\begin{array}{l}\text { Kobusingye and } \\
\text { Lett } 2000 \text { [14] }\end{array}$ & Prospective & Not mentioned & $\begin{array}{l}\text { Staff trained for } \\
1 \mathrm{~h}\end{array}$ & $\begin{array}{l}\text { One page paper } \\
\text { form then loaded } \\
\text { onto Epi Info } \\
\text { Version } 6\end{array}$ & $\begin{array}{l}\text { Crosschecked } \\
\text { with hospital } \\
\text { registration book }\end{array}$ & Moderate \\
\hline $\begin{array}{l}\text { Kobusingye et al. } \\
2002 \text { [13] }\end{array}$ & Prospective & 96.5 & $\begin{array}{l}\text { Doctors, nurses } \\
\text { and clinical } \\
\text { officers }\end{array}$ & $\begin{array}{l}\text { One page paper } \\
\text { form }\end{array}$ & $\begin{array}{l}\text { Data checked by } \\
\text { Senior Doctor }\end{array}$ & Good \\
\hline $\begin{array}{l}\text { Laing et al. } 2014 \\
\text { [17] }\end{array}$ & Prospective & 80 & $\begin{array}{l}\text { Trained } \\
\text { physicians }\end{array}$ & $\begin{array}{l}\text { Computer } \\
\text { questionnaire } \\
\text { then analysed } \\
\text { using FileMaker } \\
\text { Pro } 11\end{array}$ & Trained doctors & Good \\
\hline $\begin{array}{l}\text { Mehmood et al. } \\
2013 \text { [20] }\end{array}$ & Prospective & 97 & $\begin{array}{l}\text { Trained research } \\
\text { assistant }\end{array}$ & $\begin{array}{l}\text { Paper form then } \\
\text { analysed using } \\
\text { Karachi Trauma } \\
\text { Registry Software }\end{array}$ & $\begin{array}{l}\text { Random checks } \\
\text { of data collection } \\
\text { by Principal } \\
\text { Investigator }\end{array}$ & Good \\
\hline $\begin{array}{l}\text { Moini et al. } 2000 \\
\text { [22] }\end{array}$ & Prospective & 95 & $\begin{array}{l}\text { Trained } \\
\text { physicians }\end{array}$ & $\begin{array}{l}\text { Paper form then } \\
\text { Epi Info then } \\
\text { analysed using } \\
\text { IBM SPSS }\end{array}$ & $\begin{array}{l}\text { Trained } \\
\text { physicians }\end{array}$ & Good \\
\hline $\begin{array}{l}\text { Nottidge et al. } \\
2014 \text { [25] }\end{array}$ & Prospective & $\begin{array}{l}\text { Varied } \\
\text { completeness of } \\
\text { data collection }\end{array}$ & Not mentioned & $\begin{array}{l}\text { Paper form then } \\
\text { Epi Info }\end{array}$ & Not mentioned & Moderate-poor \\
\hline $\begin{array}{l}\text { Ordóñez et al. } \\
2012 \text { [29] }\end{array}$ & $\begin{array}{l}\text { Prospective and } \\
\text { retrospective }\end{array}$ & 37.6 & $\begin{array}{l}\text { Full time staff for } \\
\text { data recording }\end{array}$ & $\begin{array}{l}\text { International } \\
\text { Trauma Registry } \\
\text { web-based form }\end{array}$ & $\begin{array}{l}\text { Electronic } \\
\text { retrieval from } \\
\text { electronic notes }\end{array}$ & Good \\
\hline $\begin{array}{l}\text { Petroze et al. } \\
2014 \text { [32] }\end{array}$ & Prospective & Not mentioned & $\begin{array}{l}\text { Trained data } \\
\text { manager }\end{array}$ & $\begin{array}{l}\text { Paper form then } \\
\text { entered into } \\
\text { Microsoft Access }\end{array}$ & $\begin{array}{l}\text { Trained data } \\
\text { manager }\end{array}$ & Good \\
\hline $\begin{array}{l}\text { Plummer et al. } \\
2010 \text { [33] }\end{array}$ & Prospective & Not mentioned & Not mentioned & $\begin{array}{l}\text { Collected and } \\
\text { transferred to } \\
\text { Trauma! Software } \\
\text { programme }\end{array}$ & Not mentioned & Moderate-poor \\
\hline $\begin{array}{l}\text { Rabbani and } \\
\text { Moini } 2007 \text { [34] }\end{array}$ & Prospective & Not mentioned & $\begin{array}{l}\text { Trained } \\
\text { physicians }\end{array}$ & Not mentioned & $\begin{array}{l}\text { Trained } \\
\text { physicians }\end{array}$ & Moderate-poor \\
\hline $\begin{array}{l}\text { Roy et al. } 2010 \\
\text { [37] }\end{array}$ & Prospective & 95 & $\begin{array}{l}\text { Medical intern } \\
\text { collecting data }\end{array}$ & $\begin{array}{l}\text { Questionnaire } \\
\text { then analysed } \\
\text { using STATA }\end{array}$ & $\begin{array}{l}\text { Dedicated intern } \\
\text { collecting data }\end{array}$ & Good \\
\hline $\begin{array}{l}\text { Sabariah et al. } \\
2008 \text { [38] }\end{array}$ & Not mentioned & Not mentioned & Not mentioned & Not mentioned & Not mentioned & Moderate-poor \\
\hline $\begin{array}{l}\text { Samuel et al. } \\
2010 \text { [39] }\end{array}$ & Prospective & Not mentioned & $\begin{array}{l}\text { Trained registry } \\
\text { clerk } 24 \mathrm{~h} / \text { day }\end{array}$ & $\begin{array}{l}\text { Double-sided } \\
\text { registry form }\end{array}$ & $\begin{array}{l}\text { Trained registry } \\
\text { clerk } 24 \mathrm{~h} \text { /day }\end{array}$ & Moderate \\
\hline
\end{tabular}


Table 2. (continued)

\begin{tabular}{|c|c|c|c|c|c|c|}
\hline Author, Year & Data capture & $\begin{array}{c}\text { Reported } \\
\text { completeness of } \\
\text { data }(\%)\end{array}$ & $\begin{array}{c}\text { Data collection } \\
\text { staff }\end{array}$ & $\begin{array}{l}\text { Trauma data } \\
\text { collection } \\
\text { methods }\end{array}$ & $\begin{array}{l}\text { Methods to } \\
\text { optimise data } \\
\text { quality }\end{array}$ & $\begin{array}{c}\text { Overall } \\
\text { subjective } \\
\text { assessment }\end{array}$ \\
\hline $\begin{array}{l}\text { Schuurman et al. } \\
2011 \text { [40] }\end{array}$ & Prospective & $\begin{array}{l}\text { Varied: displayed } \\
\text { as a table in the } \\
\text { paper }\end{array}$ & $\begin{array}{l}\text { Two trained } \\
\text { researchers }\end{array}$ & Paper form & $\begin{array}{l}\text { Two trained } \\
\text { researchers }\end{array}$ & Good \\
\hline $\begin{array}{l}\text { Seidenberg et al. } \\
2014 \text { [41] }\end{array}$ & Prospective & Not mentioned & $\begin{array}{l}\text { Trained staff } \\
24 \text { h/day }\end{array}$ & $\begin{array}{l}\text { Registry } \\
\text { questionnaire, } \\
\text { then Cardiff } \\
\text { Teleform }\end{array}$ & $\begin{array}{l}\text { Trained staff } \\
24 \text { h/day and data } \\
\text { collected twice } \\
\text { daily when } \\
\text { admitted }\end{array}$ & Good \\
\hline $\begin{array}{l}\text { Squyer et al. } 2008 \\
\text { [42] }\end{array}$ & Retrospective & 75 & Not mentioned & $\begin{array}{l}\text { Medical records } \\
\text { reviewed from } \\
\text { trauma patients }\end{array}$ & Not mentioned & Moderate \\
\hline $\begin{array}{l}\text { Wainiqolo et al. } \\
2012 \text { [43] }\end{array}$ & Not mentioned & Not mentioned & $\begin{array}{l}\text { Research } \\
\text { assistants and } \\
\text { hospital nurses }\end{array}$ & $\begin{array}{l}\text { Injury } \\
\text { surveillance } \\
\text { questionnaire }\end{array}$ & $\begin{array}{l}\text { Research } \\
\text { assistants }\end{array}$ & Moderate \\
\hline $\begin{array}{l}\text { Ward et al. } 2010 \\
\text { [44] }\end{array}$ & $\begin{array}{l}\text { Prospective and } \\
\text { retrospective }\end{array}$ & Not mentioned & $\begin{array}{l}\text { Trained medical } \\
\text { records clerks }\end{array}$ & Not mentioned & $\begin{array}{l}\text { Trained medical } \\
\text { records clerks }\end{array}$ & Moderate \\
\hline $\begin{array}{l}\text { Zafar et al. } 2002 \\
\text { [51] }\end{array}$ & Prospective & 97 & $\begin{array}{l}\text { Trained } \\
\text { researcher }\end{array}$ & $\begin{array}{l}\text { Trauma paper } \\
\text { form then } \\
\text { electronic } \\
\text { Trauma Registry } \\
\text { v3.0 }\end{array}$ & $\begin{array}{l}\text { Trained } \\
\text { researcher }\end{array}$ & Good \\
\hline
\end{tabular}

measures in 1990 [23]. Such safety measures, in combination with road safety education, are urgently required in LMICs $[11,16]$ and the health sector should champion these measures, as recommended by the World Health Organization [35]. RTIs can be reduced by enforcing speed limits, drink-driving laws, seat-belt laws and helmet use amongst motorcyclists [3].

The need to improve road safety globally has previously been highlighted but there has been limited action taken in LMICs [12]. This led to the initiation of the "Road Safety in 10 Countries Project" being initiated in 2012 [12]. This highly promising road safety project is predicted to save 10,310 lives over 5 years [6]. Positive potential side-effects of improved road safety are an increase in walking and cycling and a reduction in pollution [2].

The young men identified by this review as most affected by trauma are also often the family breadwinners in LMICs [47] and their death or disability from injury may drive these families into poverty [48]. Similarly the cost of care for injured young men can place unsustainable demands on families, especially in the context of underdeveloped social care and security systems [50].

This systematic review utilised data from trauma registries to determine the demographics of trauma patients in LMICs. In a scoping review of world trauma registries in 2012, publications from trauma registries were identified from 35 countries with the majority of publications from the US and Australia [27]. Trauma registries can be used as part of a trauma quality improvement programme $[9,29]$. Implementation of trauma quality improvement programmes, which include trauma registries, has resulted in decreased mortality from trauma [9]. By identifying trends in injury, prevention strategies can be designed [4]. Trauma quality improvement programmes may also reduce overall hospital costs [5].

South Africa was identified to have a relatively high rate of penetrating injuries (including stabbings and gunshots). In the paper by Laing et al. they reported $40.5 \%$ of their injuries to be penetrating [17]. Jamaica also had a relatively high rate of penetrating trauma with $27.4 \%$ of the injuries in the paper by Ward et al. attributed to penetrating trauma [44]. This relatively high rate of violent trauma in these countries should be addressed by the local governments. Laing et al. discuss the fact that there is a high rate of interpersonal violence in South Africa [17] and Ward et al. explain that the "Violence Prevention Programme" was set up in Jamaica in 2004 to address the growing problem [44].

Time to hospital varied largely between countries and only a few of the trauma registry articles in this review, contained this information. In LMIC trauma registries in this review pre-hospital transfer times were long and the availability of ambulance transfers was limited. Long pre-hospital transfer times may be associated with worse outcomes [39]. This is an issue that needs addressing by local governments.

The quality of articles analysed in this review was variable. In a review by O'Reilly et al. they devised a tool to analyse data from trauma registries, which they named the "Trauma Registry Assessment Tool" [28]. This tool helps to assess the physical resources, human resources and processes of a trauma registry and is displayed in Table 1 of their paper [28]. We used the tool to assess the overall quality of articles we analysed. Table 2 displays our assessment of article quality using this assessment tool. Most articles reported prospective data but completeness of data collection was often not 
Table 3. Quantitative synthesis of systematic review.

\begin{tabular}{|c|c|c|c|c|c|c|c|c|}
\hline Author, Year & $\begin{array}{l}\text { Average age } \\
\text { (years) }\end{array}$ & $\%$ Male & $\begin{array}{c}\text { \% Road traffic } \\
\text { injuries } \\
\text { (RTIs) }\end{array}$ & $\begin{array}{c}\% \text { Stabbing } \\
\text { or gunshot }\end{array}$ & $\begin{array}{l}\% \text { Blunt } \\
\text { force }\end{array}$ & $\%$ Fall & $\begin{array}{c}\% \text { Other } \\
\text { cause }\end{array}$ & $\begin{array}{l}\text { Trauma scoring } \\
\text { system(s) used } \\
\text { (see below for } \\
\text { abbreviations) }\end{array}$ \\
\hline $\begin{array}{l}\text { Demyttenaere } \\
\text { et al. } 2009 \text { [4] }\end{array}$ & 26 (mean) & 75 & 50 & 10 & 15 & 10 & 2 & KTS \\
\hline $\begin{array}{l}\text { Haghparast- } \\
\text { Bidgoli et al. } \\
2013 \text { [8] }\end{array}$ & $\begin{array}{l}31 \text { (mean), } 26 \\
\text { (median) }\end{array}$ & 78 & 47 & $\begin{array}{c}\text { Not } \\
\text { mentioned }\end{array}$ & $\begin{array}{c}\text { Not } \\
\text { mentioned }\end{array}$ & $\begin{array}{c}\text { Not } \\
\text { mentioned }\end{array}$ & $\begin{array}{c}\text { Not } \\
\text { mentioned }\end{array}$ & GCS, ISS \\
\hline $\begin{array}{l}\text { Hashmi et al. } \\
2013 \text { [9] }\end{array}$ & $\begin{array}{c}\text { Most patients } \\
26-35\end{array}$ & 87 & 59 & 19.6 & 0 & 5.6 & 9.1 & GCS, ISS, RTS \\
\hline $\begin{array}{l}\text { Kobusingye et al. } \\
2002[13]\end{array}$ & 24 (mean) & 73 & 50 & 16 & 0 & 13 & - & KTS \\
\hline $\begin{array}{l}\text { Laing et al. } \\
2014 \text { [17] }\end{array}$ & 28 (mean) & 82 & $\begin{array}{c}\text { Not } \\
\text { documented }\end{array}$ & 40.5 & $\begin{array}{c}54.7 \\
\text { (includes } \\
\text { RTIs) }\end{array}$ & 0 & 4.8 & ISS \\
\hline $\begin{array}{l}\text { Mehmood et al. } \\
2013 \text { [20] }\end{array}$ & 27 (mean) & 72 & 33 & 7 & 0 & 37 & 16 & $\begin{array}{c}\text { GCS, ISS, RTS, } \\
\text { TRISS }\end{array}$ \\
\hline $\begin{array}{l}\text { Nottidge et al. } \\
2014 \text { [25] }\end{array}$ & $\begin{array}{c}\text { Most patients } \\
20-39\end{array}$ & 74 & "Most" & $\begin{array}{c}\text { Not } \\
\text { mentioned }\end{array}$ & $\begin{array}{c}\text { Not } \\
\text { mentioned }\end{array}$ & $\begin{array}{c}\text { Not } \\
\text { mentioned }\end{array}$ & $\begin{array}{c}\text { Not } \\
\text { mentioned }\end{array}$ & AIS. ISS \\
\hline $\begin{array}{l}\text { Ordóñez et al. } \\
2012 \text { [29] }\end{array}$ & 31 (mean) & 67 & 21 & 19.8 & 0 & 33.7 & 20.8 & GCS, ISS, RTS \\
\hline $\begin{array}{l}\text { Petroze et al. } \\
2014 \text { [32] }\end{array}$ & $\begin{array}{c}30 \text { (mean), } 27 \\
\text { (median) }\end{array}$ & 75 & 48 & 4 & 14 & 28 & 6 & GCS \\
\hline $\begin{array}{l}\text { Rabbani and } \\
\text { Moini } 2007 \text { [34] }\end{array}$ & 28 (mean) & 78 & 46 & 5.1 & 14.9 & 19 & 0 & $\begin{array}{l}\text { AIS, ASCOT, } \\
\text { ISS, TRISS }\end{array}$ \\
\hline $\begin{array}{l}\text { Roy et al. } \\
2010 \text { [37] }\end{array}$ & 30 (mean) & 84 & 46 & 0 & 0 & 17 & 29 & Not mentioned \\
\hline $\begin{array}{l}\text { Sabariah et al. } \\
2008 \text { [38] }\end{array}$ & $\begin{array}{c}\text { Most patients } \\
15-24\end{array}$ & 84 & 73 & $\begin{array}{c}\text { Not } \\
\text { mentioned }\end{array}$ & $\begin{array}{c}\text { Not } \\
\text { mentioned }\end{array}$ & $\begin{array}{c}\text { Not } \\
\text { mentioned }\end{array}$ & $\begin{array}{c}\text { Not } \\
\text { mentioned }\end{array}$ & GCS, ISS \\
\hline $\begin{array}{l}\text { Samuel et al. } \\
2010 \text { [39] }\end{array}$ & 26 (median) & 76 & 43 & 0 & 0 & 13.5 & 29.6 & Not mentioned \\
\hline $\begin{array}{l}\text { Schuurman et al. } \\
2011 \text { [40] }\end{array}$ & $\begin{array}{c}\text { Most patients } \\
20-39\end{array}$ & 75 & 22 & 22 & 16 & 0 & 0 & AIS, ISS, KTS \\
\hline $\begin{array}{l}\text { Seidenberg et al. } \\
2014 \text { [41] }\end{array}$ & 24 (median) & 72 & 26 & 3.4 & 2.7 & 26.3 & 25.8 & KTS \\
\hline $\begin{array}{l}\text { Ward et al. } \\
2010 \text { [44] }\end{array}$ & Most under 29 & 64 & 17 & 27.4 & 17 & 44 & 3.6 & Not mentioned \\
\hline
\end{tabular}

Abbreviations of trauma scores: AIS: Abbreviated Injury Scale; ASCOT: A Severity Characterization Of Trauma; GCS: Glasgow Coma Scale; ISS: Injury Severity Score; KTS: Kampala Trauma Score; RTS: Revised Trauma Score; TRISS: Trauma and Injury Severity Score.

reported. Most trauma registries initially collected data on paper forms and then transferred this information to computer. We would like to propose that trauma registries report their data in the format of the "Trauma Registry Assessment Tool". This would ensure that articles from trauma registries would be of a consistently high standard and that all important data is published. By presenting the data in this way it would allow funding bodies and governments to identify the areas of greatest need of investment and support. Trauma registries are expensive to run and therefore have an ethical obligation to publish data in an easy-to-read and consistent format so that their cost can be justified.

A limitation of this systematic review is that it only includes data from trauma registries and linked data may better estimate the age, gender and mechanism of injury in
LMIC trauma patients. Additionally, trauma registry data may suffer from a decreased capture rate of data because busy clinicians may not have the time to record every trauma episode $[13,42]$. Trauma registry data will only capture the data of injured patients who attend hospitals with trauma registries and will miss those patients who have their injuries treated in the community or die before reaching hospital $[14,40]$.

In summary, this trauma registry study has identified that the young, male population is most affected by trauma in LMICs and $46 \%$ of all injuries were road traffic injuries. This information can be used by local and national governments to support the case for increased investment in road safety measures and other strategies targeted at injury prevention in this population group. 
Table 4. Pre-hospital transfer times and methods review.

\begin{tabular}{|c|c|c|c|c|c|c|c|c|c|}
\hline Author, Year & $\begin{array}{c}\text { Pre-hospital } \\
\text { transfer (PHT) time } \\
\text { (minutes) }\end{array}$ & $\begin{array}{c}\text { Ambulance } \\
(\%)\end{array}$ & $\begin{array}{c}\text { Private } \\
\text { Vehicle } \\
(\%)\end{array}$ & $\begin{array}{c}\text { Walking } \\
(\%)\end{array}$ & $\begin{array}{c}\text { Public } \\
\text { transport } \\
(\%)\end{array}$ & $\begin{array}{c}\text { Police } \\
(\%)\end{array}$ & $\begin{array}{c}\text { Bike/ } \\
\text { motorbike } \\
(\%)\end{array}$ & $\begin{array}{l}\text { Taxi } \\
(\%)\end{array}$ & $\begin{array}{l}\text { Other } \\
(\%)\end{array}$ \\
\hline $\begin{array}{l}\text { Hsia et al. } \\
2010 \text { [11] }\end{array}$ & Not mentioned & 5 & 50 & 10 & 0 & 22 & 12 & 0 & 0 \\
\hline $\begin{array}{l}\text { Kobusingye et al. } \\
2002 \text { [13] } \\
\text { Mehmood et al. } \\
2013 \text { [20] }\end{array}$ & $\begin{array}{c}66 \% \text { patients } \\
\text { within } 60 \mathrm{~min} \\
81 \% \text { patients } \\
\text { within } 360 \mathrm{~min}\end{array}$ & - & - & - & - & - & - & - & - \\
\hline $\begin{array}{l}\text { Nottidge et al. } \\
2014 \text { [25] }\end{array}$ & Not mentioned & 0 & 98 & 0 & 0 & 0 & 0 & 0 & 2 \\
\hline $\begin{array}{l}\text { Roy et al. } \\
2010 \text { [37] }\end{array}$ & Not mentioned & 34.5 & 0 & 0 & 0 & 24.4 & 0 & 39.3 & 0 \\
\hline $\begin{array}{l}\text { Samuel et al. } \\
2010 \text { [39] }\end{array}$ & 201 & 15.4 & 43.8 & 14.5 & 12.4 & 7.8 & 0 & 0 & 0 \\
\hline $\begin{array}{l}\text { Seidenberg et al. } \\
2014 \text { [41] }\end{array}$ & 180 & 5.8 & 51.8 & 0 & 37.1 & 0 & 0 & 0 & 0 \\
\hline $\begin{array}{l}\text { Squyer et al. } \\
2008 \text { [42] }\end{array}$ & Not mentioned & 78.7 & 21.3 & 0 & 0 & 0 & 0 & 0 & 0 \\
\hline $\begin{array}{l}\text { Zafar et al. } \\
2002 \text { [51] }\end{array}$ & 98 & 6.4 & 0 & 0 & 0 & 0 & 0 & 0 & 93.5 \\
\hline
\end{tabular}

\section{Conflicts of interest}

OB and GGJ declare no conflicts of interest.

CBDL and CEG are both Lancet Commissioners on Global Surgery but declare no conflicts of interest related to this work.

Acknowledgements. The authors would like to thank Mr Tim Reeves, librarian, for his assistance with the literature search for this systematic review.

The authors would like to thank the UK National Institute for Health Research (NIHR) for funding this research.

\section{References}

1. Anon (2014) The World Bank. Data: country and lending groups. World Bank. Retrieved August 30, 2014 (http:// data.worldbank.org/about/country-and-lending-groups).

2. Bliss T, Raffo V (2013) Improving global road safety: Towards equitable and sustainable development, guidelines for country road safety engagement. World Bank. Retrieved Feb 22, 2015 (https://openknowledge.worldbank.org/handle/10986/17627).

3. Chisholm D, Naci H, Hyder AA (2012) Cost effectiveness of strategies to combat road traffic injuries in Sub-Saharan Africa and South East Asia: mathematical modelling study. BMJ 344, e612.

4. Demyttenaere SV et al. (2009) Injury in Kampala, Uganda: 6 Years Later. Can J Surg 52(5), E146-E150.

5. DiRusso $\mathrm{S}$ et al. (2001) Preparation and achievement of American college of Surgeons level I trauma verification raises hospital performance and improves patient outcome. J Trauma 51, 294-300.

6. Esperato A, Bishai D, Hyder AA (2012) Projecting the health and economic impact of road safety initiatives: a case study of a multi-country project. Traffic Inj Prev 13(Suppl 1), 82-89.
7. Gore FM et al. (2011) Global burden of disease in young people aged 10-24 years: a systematic analysis. Lancet 377(9783), 2093-2102.

8. Haghparast-Bidgoli H, Saadat S, Bogg L, Yarmohammadian MH, Hasselberg M (2013) Factors affecting hospital length of stay and hospital charges associated with road traffic-related injuries in Iran. BMC Health Serv Res 13(1), 281.

9. Hashmi ZG et al. (2013) Hospital-based trauma quality improvement initiatives: first step toward improving trauma outcomes in the developing world. J Trauma Acute Care Surg $75(1), 60-68$.

10. Higgins J, Deeks J (2008) Chapter 7: selecting studies and collecting data, in Cochrane handbook of systematic reviews of interventions. Higgins JPT, Green S, Editors. Chicester (UK), John Wiley \& Sons.

11. Hsia RY et al. (2010) Epidemiology of injuries presenting to the national hospital in Kampala, Uganda: implications for research and policy. Int J Emerg Med 3(3), 165-172.

12. Hyder AA et al. (2012) Addressing the implementation gap in global road safety: exploring features of an effective response and introducing a 10-country program. Am J Public Health 102(6), 1061-1067.

13. Kobusingye OC, Guwatudde D, Owor G, Lett RR (2002) Citywide trauma experience in Kampala, Uganda: a call for intervention. Inj Prev 8(2), 133-136.

14. Kobusingye OC, Lett RR (2000) Hospital-based trauma registries in Uganda. J Trauma 48(3), 498-502.

15. Kotagal $\mathrm{M}$ et al. (2014) Health and economic benefits of improved injury prevention and trauma care worldwide. PLoS One 9(3), e91862.

16. Lagarde E (2007) Road traffic injury is an escalating burden in Africa and deserves proportionate research efforts. PLoS Med 4(6), e170.

17. Laing GL, Bruce JL, Aldous C, Clarke DL (2014) The design, construction and implementation of a computerised trauma registry in a developing South African metropolitan trauma service. Injury 45(1), 3-8. 
18. Lozano R et al. (2012) Global and regional mortality from 235 causes of death for 20 age groups in 1990 and 2010: a systematic analysis for the Global Burden of Disease Study 2010. Lancet 380(9859), 2095-2128.

19. Mathers CD, Loncar D (2006) Projections of global mortality and burden of disease from 2002 to 2030. PLoS Med 3(11), e442.

20. Mehmood A, Razzak JA, Kabir S, Mackenzie EJ, Hyder AA (2013) Development and pilot implementation of a locally developed trauma registry: lessons learnt in a low-income country. BMC Emerg Med 13(1), 4.

21. Moher D, Liberati A, Tetzlaff J, Altman DG (2010) Preferred reporting items for systematic reviews and meta-analyses: the PRISMA statement. Int J Surg 8(5), 336-341.

22. Moini M, Rezaishiraz H, Zafarghandi MR (2000) characteristics and outcome of injured patients treated in urban trauma centers in Iran. J Trauma 48(3), 503-507.

23. Murray CJL et al. (2012) Disability-adjusted life years (DALYs) for 291 diseases and injuries in 21 regions, 1990-2010: a systematic analysis for the Global Burden of Disease Study 2010. Lancet 380(9859), 2197-2223.

24. Norton R, Kobusingye O (2013) Injuries. NEJM 368(18), 1723-1730.

25. Nottidge TE, Dim M, Udoinyang CI, Udoh IA (2014) The Uyo Trauma Registry-developed for sustainable audit of trauma care and cause in Nigeria. Trop Doct 44(1), 14-18.

26. Nwomeh BC, Lowell W, Kable R, Haley K, Ameh EA (2006) History and development of trauma registry: lessons from developed to developing countries. World J Emerg Surg 1, 32.

27. O'Reilly GM, Cameron PA, Joshipura M (2012) Global trauma registry mapping: a scoping review. Injury 43(7), 1148-1153.

28. O'Reilly GM, Joshipura M, Cameron PA, Gruen R (2013) Trauma registries in developing countries: a review of the published experience. Injury 44(6), 713-721.

29. Ordóñez CA et al. (2012) Experience of two first level hospitals in the southwest region of Colombia on the implementation of the Panamerican Trauma Society International Trauma Registry. Rev Col Bras Cir 39(4), 255-262.

30. Patton GC et al. (2009) Global patterns of mortality in young people: a systematic analysis of population health data. Lancet 374(9693), 881-892.

31. Peden M, McGee K, Krug E (2002) Injury: a leading cause of the global burden of disease, 2000. Geneva, World Health Organization.

32. Petroze RT et al. (2014) Infectious outcomes assessment for health system strengthening in low-resource settings: the novel use of a trauma registry in Rwanda. Surg Infect 15(4), 382-386.

33. Plummer J et al. (2010) Trauma: the burden of a preventable problem trauma. West Indian Med J 59(1), 26-28.

34. Rabbani A, Moini M (2007) Application of "Trauma and Injury Severity Score" and "A Severity Characterization of Trauma" score to trauma patients in a setting different from "Major Trauma Outcome Study". Arch Iran Med 10(3), 383-386.
35. Racioppi F, Eriksson L, Tingvall C, Villaveces A (2004) Preventing road traffic injury: a public health perspective for Europe. WHO Retrieved July 15, 2014 (http://www.euro. who.int/_data/assets/pdf_file/0003/87564/E82659.pdf).

36. Raffo V, Bliss T, Shotten M, Sleet D, Blanchard C (2013) Case study: The Argentina Road Safety Project: lessons learned for the decade of action for road safety, 2011-2020. Glob Health Promot 20(4 Suppl), 20-36.

37. Roy $\mathrm{N}$ et al. (2010) Where there are no emergency medical services-prehospital care for the injured in Mumbai, India. Prehosp Disaster Med 25(2), 145-151.

38. Sabariah F, Ramesh N, Mahathar A (2008) National Trauma Database (NTrD) - improving trauma care: first year report. Med J Malaysia 63, 45-49.

39. Samuel JC et al. (2010) Hospital-based injury data in Malawi: strategies for data collection and feasibility of trauma scoring tools. Trop Doct 40(2), 98-99.

40. Schuurman $\mathrm{N}$ et al. (2011) collecting injury surveillance data in low- and middle-income countries: the Cape Town Trauma Registry pilot. Glob Public Hlth 6(8), 874-889.

41. Seidenberg P et al. (2014) epidemiology of injuries, outcomes, and hospital resource utilisation at a tertiary teaching hospital in Lusaka, Zambia. Afr J Emerg Med 4(3), 115-122.

42. Squyer E et al. (2008) comparison of trauma mortality between two hospitals in Turkey to one trauma center in the US. Eur J Emerg Med 15(4), 209-213.

43. Wainiqolo I et al. (2012) A profile of injury in Fiji: findings from a population-based injury surveillance system (TRIP-10). BMC Publ Health 12(1), 1074.

44. Ward E et al. (2010) The Jamaica Injury Surveillance System: a profile of the intentional and unintentional injuries in Jamaican hospitals. West Indian Med J 59(876), 7-13.

45. World Bank (2014) World Bank. Roads \& HIghways: Road Safety. Retrieved August 30, 2014 (http://www.worldbank.org/ transport/roads/safety.htm).

46. World Health Organization (1996) Investing in Health Research and Development. Retrieved Aug 20, 2014 (http://whqlibdoc. who.int/hq/1996/TDR_GEN_96.2.pdf).

47. World Health Organization (2004) World report on road traffic injury prevention. Geneva, World Health Organization. Retrieved July 15, 2014 (http://www.who.int/violence_injury_ prevention/publications/road_traffic/world_report/summary_en_ rev.pdf).

48. World Health Organization (2009) Global status report on road safety: time for action. Geneva, World Health Organization. Retrieved July 28, 2014 (www.who.int/violence_).

49. World Health Organization (2010) Injuries and violence: the facts. Retrieved July 25, 2014 (http://whqlibdoc.who.int/ publications/2010/9789241599375_eng.pdf).

50. Young S (2014) Orthopaedic trauma surgery in low-income countries. Follow-up, infections and HIV. Acta Orthop 85(S356), 1-32.

51. Zafar H, Rehmani R, Raja AJ, Ali A, Ahmed M (2002) Registry based trauma outcome: perspective of a developing country. Emerg Med J 19(5), 391-394.

Cite this article as: Boughton O, Jones GG, Lavy CBD \& Grimes CE (2015) Young, male, road traffic victims: a systematic review of the published trauma registry literature from low and middle income countries. SICOT J, 1, 10 